\title{
PAH under XUV excitation: an ultrafast XUV- photochemistry experiment for astrophysics
}

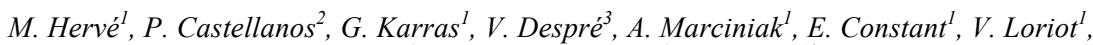 \\ A.I. Kuleff ${ }^{3}, A$. G. G. M. Tielens ${ }^{2}, F$. Lépine ${ }^{1 *}$ \\ ${ }^{1}$ University of Lyon, Université Claude Bernard Lyon 1, CNRS, Institut Lumière Matière, F-69622 \\ Villeurbanne, France \\ ${ }^{2}$ Observatory, Leiden University, P.O. Box 9513, 2300 RA Leiden, The Netherlands \\ ${ }^{3}$ Physikalisch-Chemisches Institut, Universität Heidelberg, Im Neuenheimer Feld 229, Heidelberg \\ 69120, Germany
}

Corresponding Author e-mail address: franck.lepine@univ-lyon1.fr

\begin{abstract}
Understanding processes induced by XUV excitation of Polycyclic Aromatic Hydrocarbons (PAHs) is at the heart of molecular astrophysics, which aims at understanding molecular evolution in interstellar media. We used ultrashort XUV pulses to produce highly excited PAHs cations. The photo-induced dynamics is probed using a pump-probe XUV-IR spectroscopy. By studying PAH from small (naphthalene) to large (hexabenzocoronene) PAHs, we show that the dynamic is governed by the large density of states, in which many-body quantum effects are dominant.
\end{abstract}

\section{Introduction}

The advent of sources producing ultrashort XUV pulses has brought new perspectives in observing atoms and molecules on their natural time scale. First experiments have been performed on molecules showing the ability to observe and control electron and (coupled) nuclear dynamics in real time, down to the attosecond timescale $[1,2]$. In this context, polycyclic aromatic hydrocarbons (PAHs) are rigid and symmetric molecules and therefore constitute prototypes for the investigation of large and complex systems. Moreover, due to their high stability under energetic radiation, they are even thought as a primordial class of large molecules in space [3], where their photo-induced reactions are of prior importance to comprehend the appearance of biomolecules in interstellar chemistry. In order to investigate the photoinduced processes upon XUV excitation, we have recorded fragmentation and ionization dynamics in PAHs of various sizes, from Naphthalene $\left(\mathrm{C}_{10} \mathrm{H}_{8}\right)$ to Hexabenzocoronene (HBC, $\mathrm{C}_{42} \mathrm{H}_{18}$ ) using XUV-IR pump-probe scheme.

\section{XUV-IR probe experiments}

Our experimental set-up is based on the combination of an XUV pump-IR probe scheme and a Velocity Map Imaging spectrometer operating either in time-of-flight or momentum imaging mode [4,5]. Following XUV ionization, PAH cations are formed in highly excited states. Consequently, non-adiabatic couplings between electronic states determine their ultrafast relaxation. In addition, electron correlation also plays a crucial role and prevails for the states lying around the second ionization potential [6]. In order to monitor the dynamics of the states, we use a second time-delayed, IR pulse from which the molecule 
can absorb photons. This absorption leads either to a second ionization step that produces stable dications or fragments from the cation. We have measured the variation of the molecular ions and fragments yields as a function of the pump-probe delay (see Fig. 1).

\section{Dynamics of highly excited PAHs}

\subsection{Dynamics in the dications}

For all the investigated PAHs, the dication signal in Fig 1.a reveals an ultrafast transient signal with a direct population of excited states, followed by a fast decay $(40 \pm 5$ fs for Pyrene, $49 \pm 9$ fs for Coronene, and $59 \pm 10$ fs for HBC). This shows that the measured timescale increases with the size of the molecule (see also Fig 2.a). Guided by quantum mechanical calculations, we have interpreted this behaviour is terms of a complex relaxation through a dense population of shake-up states. Considering states close to the double ionization threshold, computed ionization spectra (Fig 2.b) confirmed that these states have a strong multielectronic character, and are also coupled by non BornOppenheimer effects. Increasing the size of the molecule leads to a higher density of strongly non-adiabatically coupled shake-up states. As a consequence, the reported trend of the relaxation dynamics corresponds to the fact that the initial population has to cross a large and increasingly number of conical intersections for larger and larger molecules, leading to the few tens of fs timescale measured. Therefore, this mechanism seems general for the dynamics of highly excited PAHs. We have also validated this scenario by performing time-resolved electron spectroscopy experiments [7] where we have shown that higher energy states have longer lifetime due to the increase of the density of shake-ups.

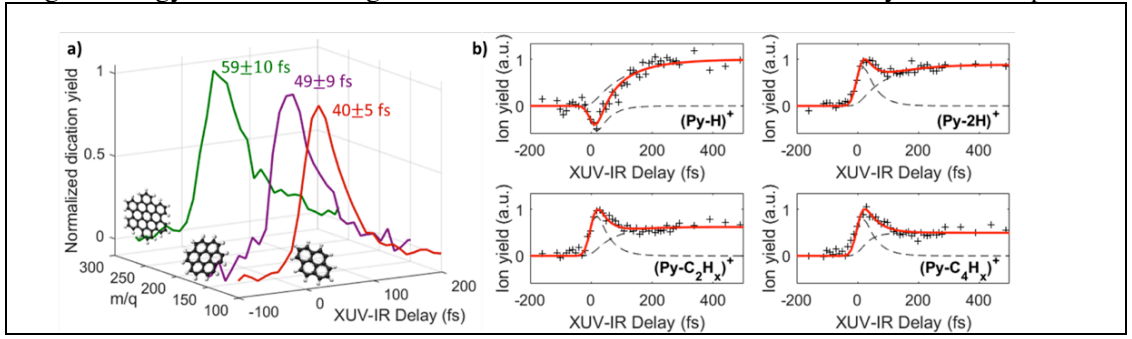

Fig. 1. a) Time-dependent dication yield measured for three studied large PAHs: Pyrene $\left(\mathrm{C}_{16} \mathrm{H}_{10}\right)$, Coronene $\left(\mathrm{C}_{24} \mathrm{H}_{12}\right)$, $\mathrm{HBC}$; b) Time-dependent fragment yield for Pyrene: loss of $\mathrm{H}, 2 \mathrm{H}, \mathrm{C}_{2} \mathrm{Hx}$ and $\mathrm{C}_{4} \mathrm{H}_{\mathrm{x}}$ are presented.

\subsection{Dynamics in the fragments}

We pushed the experiment one step further by observing the time-dependent fragment yield. The usual fragmentation pattern corresponds to the loss of one or several hydrogen atoms, and the loss of $\mathrm{C}_{2} \mathrm{H}_{2}$. Observation of the fragment yields has revealed a population of fragmentation channels on a $100 \mathrm{fs}$ timescale (for instance, 119 $\pm 26 \mathrm{fs}$ for $\mathrm{H}$ loss and $105 \pm 30$ fs for $2 \mathrm{H}$ loss in the case of Pyrene (Fig 1.b)). We interpret this dynamics as the vibrational energy spreads in the molecule when the population is transferred from one high lying electronic state to a lower one. If the total energy absorbed is higher than the fragmentation threshold of the cation, this dynamics manifests itself as a progressive increase of the fragmentation yield. For higher-mass fragments, this effect is combined with the decay of the multielectronic states mentioned above. Indeed, the high internal energy required for the loss of high-mass fragments implies that the absorption of a IR photon can either lead to second ionization, forming a stable or unstable dication that can fragment. 

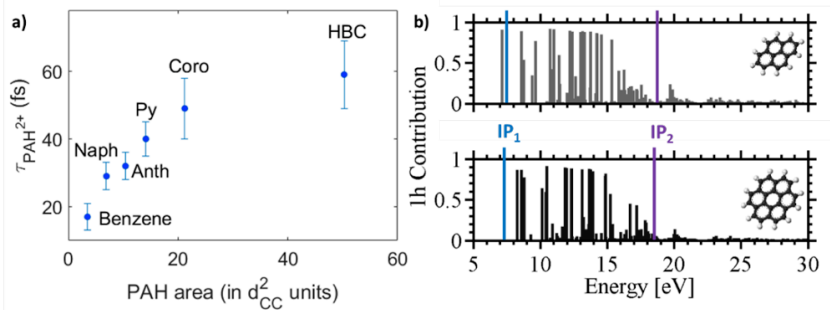

Fig. 2. a) Dication decay constant as a function of the PAH area (given in units of the distance between two adjacent carbon atoms, $\mathrm{d}_{\mathrm{CC}}$ ); Benzene $(\mathrm{C} 6 \mathrm{H} 6)$ is taken from [8]; b) Computed ADC spectra for Pyrene and Coronene, from Supplementary Material of [6].

\section{Conclusion}

This study reports dynamics occurring in small and large PAHs (up to 60 atoms) after XUV absorption. This dynamics is driven by the complex interplay between multielectronic and non-adiabatic effects. Counter intuitively, the lifetime of the relaxation dynamics increases with the molecular size. This is understood in terms of a dense shake-up structure in which the population is efficiently trapped. This general trend would allow predicting any PAH lifetimes and can be incorporated in astrochemisry models.

\section{References}

1. G. Sansone et al, "Electron Localization Following Attosecond Molecular Photoionization”, Nature 465, 763-767 (2010).

2. F. Lépine, M. Y. Ivanov, M.J.J. Vrakking, “Attosecond Molecular Dynamics: Fact or Fiction?", Nature Photonics 8, 195-204 (2014).

3. L. T. A.G.G.M Tielens, "Interstellar Polycyclic Aromatic Hydrocarbon Molecules", Ann. Rev. Astron. Astrophys. 46, 289 (2008).

4. V. Loriot, A. Marciniak, L. Quintard, V. Despré, B. Schindler, I. Compagnon, B. Concina, G. Celep, C. Bordas, F. Catoire, E. Constant, F. Lépine, "Resolving XUV Induced Femtosecond an Attosecond Dynamics in Polyatomic Molecules with a Compact Attosecond Beamline", J. Phys.: Conf. Ser. 635, 012006.

5. V. Loriot, L. Quintard, G. Karras, A. Marciniak, F. Catoire, M. Hervé, I. Compagnon, G. Renois-Predelus, B. Schnidler, B. Concina, G. Celep, R. Brédy, C. Bordas, F. Lépine and E. Constant, «Time-resolved and spectrally resolved ionization with a single ultrashort XUV-IR beamline » J. Phys. B: At. Mol. Opt. Phys. 35 A67-A74 (2018).

6. A. Marciniak, V. Despré, T. Barillot, A. Rouzée, M.C.E. Galbraith, J. Klei, C.-H. Yang, C.T.L. Smeenk, V. Loriot, S. Nagaprasad Reddy, A.G.G.M Tielens, S. Mahapatra, A.I. Kuleff, M.J.J. Vrakking, F. Lépine, "XUV Excitation Followed by Ultrafast Non-adiabatic Relaxation in PAH Molecules as a Femto-astrochemistry Experiment", Nat. Commun. 6, 7909 (2015).

7. A. Marciniak et al. (submitted) (2018).

8. M.C.E. Galbraith, C.T.L. Smeenk, G. Reitsma, A. Marciniak, V. Despré, J. Mikosch, N. Zhavoronkov, M.J.J. Vrakking, O. Kornilov, F. Lépine, "XUV-induced Reactions in Benzene on Sub-10 fs Timescale: Nonadiabatic Relaxation and Proton Migration", Phys. Chem. Chem. Phys. 19, 19822 (2017). 